\title{
Hypothermic machine perfusion attenuates ischemia/reperfusion injury against rat livers donated after cardiac death by activating the Keap1/Nrf2-ARE signaling pathway
}

\author{
SHUAI XUE ${ }^{1}$, WEIYANG HE ${ }^{1}$, XIANPENG ZENG ${ }^{1}$, ZIMEI TANG $^{1}$, \\ SHOUCHENG FENG ${ }^{1}$, ZIBIAO ZHONG ${ }^{1}$, YAN XIONG ${ }^{1}$, YANFENG WANG $^{1}$ and QIFA YE $^{1,2}$ \\ ${ }^{1}$ Zhongnan Hospital of Wuhan University, Institute of Hepatobiliary Diseases of Wuhan University, \\ Transplant Center of Wuhan University, Hubei Key Laboratory of Medical Technology on Transplantation, Wuhan, \\ Hubei 430071; ${ }^{2}$ Research Center of National Health Ministry on Transplantation Medicine, Engineering and Technology, \\ The Third Xiangya Hospital of Central South University, Changsha, Hunan 410013, P.R. China
}

Received December 6, 2017; Accepted May 4, 2018

DOI: $10.3892 / \mathrm{mmr} .2018 .9065$

\begin{abstract}
Hypothermic machine perfusion (HMP) has been demonstrated to be a more effective method for preserving livers donated after circulatory death (DCD) than cold storage (CS); however, the underlying mechanisms remain unclear. The aim of the present study was to investigate the protective effects of HMP on rat DCD livers and the possible role of the nuclear factor erythroid 2-related factor 2 (Nrf2)/antioxidant response element (ARE) signaling pathway. A total of 18 adult male rats were randomly divided into three groups: Control, HMP and CS ( $n=6$ per group). To simulate the conditions of DCD liver transplantation, rat livers in the CS and HMP groups were subjected to $30 \mathrm{~min}$ warm ischemia following cardiac arrest and were then preserved by CS or HMP for $3 \mathrm{~h}$. Subsequently, after $1 \mathrm{~h}$ of isolated reperfusion, the extent of ischemia/reperfusion injury (IRI) and cellular functions were assessed. During reperfusion, intrahepatic resistance and bile production were measured, and the perfusion fluid was collected for liver enzyme analysis. The liver tissues were then harvested for the assessment of malondialdehyde (MDA) production, superoxide dismutase (SOD) activity, ATP levels, as well as for histological analysis, immunohistochemistry and a terminal deoxynucleotidyl transferase dUTP nick end labeling assay. Finally, the expression levels of the components associated with the Nrf2-ARE signaling pathway were
\end{abstract}

Correspondence to: Dr Shuai Xue, Zhongnan Hospital of Wuhan University, Institute of Hepatobiliary Diseases of Wuhan University, Transplant Center of Wuhan University, Hubei Key Laboratory of Medical Technology on Transplantation, 169 Donghu Road, Wuhan, Hubei 430071, P.R. China

E-mail: yqf_china@163.com

Key words: hypothermic machine perfusion, cold storage, rat livers donated after cardiac death, ischemia-reperfusion injury, anti-oxidant, nuclear factor erythroid 2-related factor 2 analyzed via western blotting and reverse transcription-quantitative polymerase chain reaction. The results of the present study revealed that, compared with in the CS group, the HMP group exhibited higher levels of ATP, bile production and SOD activity, and improved histological results; however, lower levels of liver enzymes, apoptosis and MDA were detected. Additionally, the findings of the present study also suggested that the Nrf2-ARE signaling pathway may be activated by the steady laminar flow of HMP. In conclusion, HMP may attenuate ischemia-reperfusion injury to rat DCD livers via activation of the Nrf2-ARE signaling pathway.

\section{Introduction}

Liver transplantation (LT) is the only effective means of treating end-stage liver disease; however, advances in liver transplantation are restricted by the shortage of donors (1). Numerous livers donated after cardiac death (DCD) are used in clinical practice $(2,3)$, however DCD livers are subject to prolonged warm ischemic injury, which is detrimental to liver function and may affect prognosis following transplantation (4-7). Therefore, further study into improving the quality of the DCD livers is required. Increasing evidence in liver transplant research has suggested that hypothermic machine perfusion (HMP) may be more beneficial for the quality of DCD livers than cold storage (CS) $(8,9)$; however, the specific mechanism underlying HMP requires further investigation.

Nuclear factor erythroid 2-related factor 2 (Nrf2) is a transcription factor associated with various intracellular signaling pathways and is a sensor of oxidative stress; thus, Nrf2 serves an important role in the main defense mechanisms induced by cellular oxidative stress $(10,11)$. Under physiological conditions, Nrf2 can achieve a stable balance in combination with Kelch-like ECH-associated protein-1 (Keap1), which can mediate the ubiquitination of the Nrf2/Keap1 complex (12-14). Oxidative and/or electrophilic stimuli may induce the dissociation of Nrf2 from Keap1. Nrf2 can then rapidly translocate to the nucleus to function as a strong transcriptional activator of the antioxidant response element (ARE) regulating the 
transcription of genes, including heme oxygenase-1 (HO-1), glutathione-S-transferase-1 (GST-1), NAD(P)H:quinine oxidoreductase 1 (NQO1) and glutamate cysteine ligase (GCL) (15). Numerous studies have demonstrated that the Nrf2-ARE signaling pathway serves an important role in ischemia-reperfusion injury (IRI) (16-18). In addition, Nrf2 can be activated by steady laminar flow $(19,20)$. Based on previous research (16-21), the present study hypothesized that HMP may increase the transcription of ARE-response genes via the binding of $\mathrm{Nrf} 2$, and thus reduce IRI to DCD livers by attenuating oxidative stress; the underlying mechanism was also investigated in the present study.

\section{Materials and methods}

Ethics statement. The present study was conducted according to the Experimental Animal Regulations of the People's Republic of China and the Guide for the Care and Use of Laboratory Animals of the National Institutes of Health and the Guide for the Care and Use of Laboratory Animals of the USA (22), ensuring that all animals received humane care. The present study was approved by the Ethics Committee of Wuhan University (Wuhan, China).

Animals and experimental design. A total of 18 adult male Sprague-Dawley rats (age, 8 weeks; body weight, 250 $\pm 10 \mathrm{~g}$ ) were purchased from the Experimental Animal Culture Center of Hubei Centers for Disease Control (Hubei, China), and were maintained in the Animal Experimental Center of Zhongnan Hospital of Wuhan University (Wuhan, China). The rats were fed standard chow and water, and housed under standard experimental conditions (temperature: $20-25^{\circ} \mathrm{C}$, humidity: $50-70 \%$ ) under a $12 \mathrm{~h}$ light/dark cycle. To simulate DCD liver transplantation, $30 \mathrm{~min}$ of warm ischemia was conducted in livers ( $\mathrm{n}=12$, CS and HMP groups) in situ, followed by a rewarming period of $15 \mathrm{~min}$ prior to being connected to the isolated perfused rat liver device (IPRL). To investigate the protective effects of HMP on the DCD livers, the extent of graft injury in healthy livers (non-DCD, no IRI) was compared with DCD livers preserved by either CS or HMP (described below). Following graft preservation, to simulate the period of rewarming during re-implantation, all the livers were left untouched on a petri dish at room temperature for $\sim 15 \mathrm{~min}$ prior to reperfusion (23).

Then, in order to analyze reperfusion injury, livers were re-perfused in the isolated perfusion rat liver model for $1 \mathrm{~h}$ following graft preservation within the CS and HMP groups, and the same is true for the control group. For this purpose, the following experimental groups were selected: i) Control group $(n=6)$, livers without warm ischemia and subsequent underwent $1 \mathrm{~h}$ reperfusion prior to sample collection; ii) CS group ( $\mathrm{n}=6)$, DCD livers that underwent $\mathrm{CS}$ for $3 \mathrm{~h}$, followed by $1 \mathrm{~h}$ reperfusion in vitro and the iii) HMP group $(n=6)$, in which DCD livers were connected to the HMP system. HMP was performed via the portal vein for $3 \mathrm{~h}$, followed by $1 \mathrm{~h}$ reperfusion in vitro.

Modeling procedure. Rats were anesthetized via an intraperitoneal injection of $1 \%$ sodium pentobarbital $(30 \mathrm{mg} / \mathrm{kg}$; Sinopharm Chemical Reagent Co., Ltd., Shanghai, China).
A midline incision was conducted to provide entry into the abdominal cavity. The liver was carefully separated from the attached round ligament. Subsequently, the common bile duct was cannulated using a guided epidural tube (Jiangsu Changfeng Medical Industry, Co., Ltd. (Jiangsu, China) to collect bile during reperfusion. In the experimental groups, DCD was induced by hypoxia via an incision of the diaphragm without portal clamping prior to heparinization, as described below. The onset of in situ-warm ischemia was determined to be the point of the cardiac arrest, which was maintained for $30 \mathrm{~min}$ at $29 \pm 1.5^{\circ} \mathrm{C}$. The control group did not experience the warm ischemia and the other operations were the same as the experimental groups. Subsequently, the hepatic artery was ligated and then $2 \mathrm{ml}$ saline containing 100 IU heparin (Nanjing Jiancheng Bioengineering Institute, Nanjing, China) was injected via the right iliac vein. A total of $20 \mathrm{ml}\left(4^{\circ} \mathrm{C}\right)$ histidine-tryptophan-ketoglutarate (HTK; Dr. Franz Köhler Chemie GmbH, Bensheim, Germany) solution was used to rinse the liver via the portal vein, which was cannulated using a self-made polyethylene (PE) catheter (outer diameter, $2.1 \mathrm{~mm}$, inner diameter, $1.8 \mathrm{~mm}$ ) (24). Finally, the liver was harvested and a PE catheter (internal diameter, $3 \mathrm{~mm}$ ) was used to cannulate the suprahepatic inferior vena cava for collection of hepatic effluent.

CS model establishment. Following the modeling procedure, the liver was stored in the HTK solution at $0-4^{\circ} \mathrm{C}$ for $3 \mathrm{~h}$ to maintain a static state without any treatment. Following preservation, the CS group was simulated the period of rewarming for a $15 \mathrm{~min}$ and then connected to the IPRL, which is the reperfusion device used to assessment of IRI severity against livers (described below).

HMP and the IPRL model system. HMP and IPRL was performed as previously described $(25,26)$ with certain modifications. The HTK solution served as a perfusate for HMP; the devices employed for perfusion were maintained in an ice water solution $\left(0-4^{\circ} \mathrm{C}\right)$. Following collection of the livers, the samples from the HMP group were connected to the perfusion device and perfused via the portal vein. The perfusion flow was maintained at $0.23 \mathrm{ml} / \mathrm{min} / \mathrm{g}$ (23). The perfusate was oxygenated with air and recirculated for $3 \mathrm{~h}$ (perfusate volume, $100 \mathrm{ml}$ ). Following preservation, the caudate lobe of the rat livers of the CS and HMP groups was ligated and harvested to obtain samples prior to reperfusion. The remaining rat liver tissues in the three groups were then weighed and connected via the portal vein to the IPRL system for reperfusion. IPRL is an in vitro system that simulates physiological and pathophysiological conditions of reperfusion in liver transplantation and is often used as a tool for the assessment of IRI severity against livers (27).

The same perfusion device was used for HMP, as well as for the $1 \mathrm{~h}$ reperfusion period ( $\mathrm{n}=12, \mathrm{CS}$ and HMP groups). A detailed description of the HMP and IPRL system is given in as described in a previous study (26). Krebs-Henseleit buffer (Macgene $^{\text {TM }}$ M\&C Genetechnolgy, Beijing, China) with 4\% dextran was used as a perfusate for reperfusion (28). The temperature of the perfusate was maintained at $36-37^{\circ} \mathrm{C}$ during reperfusion and oxygenated to maintain $\mathrm{PO}_{2}>500 \mathrm{mmHg}$ under the effect of mixed gas $(95 \%$ oxygen and $5 \%$ carbon 
dioxide). The flow of portal venous perfusion was maintained at $3 \mathrm{ml} / \mathrm{min} / \mathrm{g} \mathrm{(29)}$ and recirculated for $1 \mathrm{~h}$ (perfusate volume, $250 \mathrm{ml})$.

Assessment of IRI using the IPRL system. During reperfusion, which was performed for $1 \mathrm{~h}$, intrahepatic resistance (IHR) was recorded by the portal pressure and portal flow and the perfusate was collected per $15 \mathrm{~min}$. Intrahepatic resistance was calculated according to the following formula: Intrahepatic resistance $(\mathrm{mmHg} / \mathrm{ml} / \mathrm{min} / \mathrm{g}$ liver $)=$ portal pressure $(10.3 \mathrm{~mm} \mathrm{Hg}) /$ portal flow $(\mathrm{ml} / \mathrm{min} / \mathrm{g}$ liver) (30). Hepatic effluent was obtained from the perfusion fluid via the PE catheter every $15 \mathrm{~min}$. Samples were centrifuged at 14,000 $\mathrm{x} g$ and $4^{\circ} \mathrm{C}$ for 5-10 min and the supernatant was collected and stored at $-80^{\circ} \mathrm{C}$ prior to the determination of aspartate aminotransferase (AST) and alanine aminotransferase (ALT) activities. The enzyme activities were assessed using ALT (cat. no. C009-2), AST (cat. no. C010-2) and LDH assay kits (cat. no. A020-2; all Nanjing Jiancheng Bioengineering Institute), according to the manufacturer's protocol. As the density of bile was equal to the water $(23,31)$, bile production was measured at 60 min by weighing the guided epidural tube in which bile was collected from the common bile duct. Then, the bile flow was gravimetrically estimated and expressed as $\mu \mathrm{l} / \mathrm{h} / \mathrm{g}$ liver (26).

Superoxide dismutase (SOD) activity and malondialdehyde (MDA) content. Frozen liver samples were lysed with $0.05 \mathrm{M}$ Tris. $\mathrm{HCl}$ (Beijing Biotopped Science \& Technology Co., Ltd., Being, China) extraction buffer on ice. The cell lysates were centrifuged at $4^{\circ} \mathrm{C} 12,000 \mathrm{x}$ g for $10 \mathrm{~min}$. The resulting cell lysates were used to assess the SOD activity and MDA content. To measure total SOD activity, a SOD kit (Nanjing Jiancheng Bioengineering Institute, Nanjing, China) was employed according to the manufacturer's protocols. MDA content was assessed with an MDA assay kit (Nanjing Jiancheng Bioengineering Institute) based on the products of membrane lipid peroxidation, which are important indicators of oxidative damage during hepatic IRI.

ATP extraction and measurement. The hepatic concentration of ATP served as an indicator of the energy status of grafts following $1 \mathrm{~h}$ of ex situ reperfusion. The ATP content of the tissue was measured using an ATP assay kit (Nanjing Jiancheng Bioengineering Institute) according to the manufacturer's protocols. ATP levels were expressed as $\mu \mathrm{mol} / \mathrm{g}$ protein.

Histological analysis. Following reperfusion, about $0.25 \mathrm{~g}$ liver samples were fixed with $10 \%$ buffered formalin for $24 \mathrm{~h}$ at room temperature ( $\mathrm{pH}=7.2$; cat. no. G2161; Beijing Solarbio Science \& Technology, Co., Ltd., Beijing, China), embedded in paraffin and cut into $5-\mu \mathrm{m}$ sections for histological analysis via hematoxylin-eosin staining (H\&E; hematoxylin staining for 5-15 min and eosin staining for 1-3 min; all performed at room temperature). Sections were analyzed under a confocal microscope (magnification, x200; Nikon A1R/A1; Nikon Corporation, Tokyo, Japan) and images were obtained. A total of 6 fields of view per section were randomly selected for the assessment of liver damage. Numerical assessment of liver damage was conducted according to the histological criteria for assessment of liver damage (32).
Immunohistochemistry of Nrf2. Following reperfusion, a portion of the livers were fixed with $10 \%$ buffered formalin for $24 \mathrm{~h}$ at room temperature $(\mathrm{pH}=7.2$; cat. no. G2161; Beijing Solarbio Science \& Technology, Co., Ltd., Beijing, China), and then embedded in paraffin, sliced, dewaxed (Dewaxing was routinely performed at $60^{\circ} \mathrm{C}$ for $20 \mathrm{~min}$, and immediately xylene 1-3 for $10 \mathrm{~min}$, respectively. However certain sections prepared on the day could be treated at $60^{\circ} \mathrm{C}$ for $3-4 \mathrm{~h}$ ), and hydrated conventionally using an ethanol gradient (from high to low). The tissues were cut into $4-\mu \mathrm{m}$ sections and mounted on glass slides. After $30 \mathrm{~min}$ of blocking at room temperature with $5 \%$ bovine serum albumin (Beijing Solarbio Technology Co., Ltd., Beijing, China), the samples were incubated with rabbit anti-Nrf2 antibody (1:1,000; cat. no. 16396-1-AP; Wuhan Sanying Biotechnology, Wuhan, China) overnight at $4^{\circ} \mathrm{C}$. The samples were then incubated for $1 \mathrm{~h}$ at room temperature with a horseradish peroxidase-conjugated goat anti-rabbit IgG (1:3,000; cat. no. GB23303; Wuhan Goodbio Technology Co., Ltd., Wuhan, China). Subsequently, the samples were incubated with 3,3'-diaminobenzidine chromogen (DAB; Maixin-Bio Ltd.) at room temperature for 5 min, and then blocked on a coverslip. Any brown and yellow staining was considered to indicate positive Nrf2 expression, as visualized under a light microscope (magnification, x200, Leica DM2000; Leica Microsystems GmbH, Wetzlar, Germany). A total of 5 visual fields were randomly selected for analysis.

Reverse transcription-quantitative polymerase chain reaction $(R T-q P C R)$. Total RNA was isolated from the liver specimens using TRIzol ${ }^{\circledR}$ reagent (Thermo Fisher Scientific Inc., Waltham, MA, USA) according to the manufacturer's protocols. RNA was reverse transcribed into cDNA using the Easy Script One-Step gDNA Removal and cDNA Synthesis Super Mix (Beijing TransGen Biotech, Co., Ltd., Beijing, China). Total RNA (1 $\mu \mathrm{g})$, Random primer $(0.1 \mu \mathrm{g} / \mu \mathrm{l}), 2 \mathrm{X}$ ES Reaction Mix (10 $\mu \mathrm{l})$, RI Enzyme Mix (1 $\mu \mathrm{l})$, gDNA Remover (1 $\mu \mathrm{l}$ ) were employed; the solution was made up to $20 \mu \mathrm{l}$ with water (RNase-free). RT reactions were performed under the following conditions: $42^{\circ} \mathrm{C}$ for $1 \mathrm{~h}$ and $75^{\circ} \mathrm{C}$ for $5 \mathrm{~min}$. The primers were synthesized by Shanghai ShineGene Molecular Biotech, Inc. (Shanghai, China; Table I). qPCR analysis was performed with the SYBR ${ }^{\circledR}$ Select Master Mix (Applied Biosystems; Thermo Fisher Scientific, Inc.) in a StepOnePlus Real-Time PCR system (Applied Biosystems; Thermo Fisher Scientific, Inc.) to determine the mRNA expression levels of Nrf2, HO-1, NQO1, GST-1, GCL and $\beta$-actin. The thermocycling conditions were as follows: $50^{\circ} \mathrm{C}$ for $2 \mathrm{~min}, 95^{\circ} \mathrm{C}$ for $10 \mathrm{~min}$, followed by 40 cycles of $95^{\circ} \mathrm{C}$ for $10 \mathrm{sec}$ and $60^{\circ} \mathrm{C}$ for $30 \mathrm{sec}$. Expression levels were normalized to $\beta$-actin, which was measured on the same plate; the differences were calculated via the $2^{-\Delta \Delta \mathrm{Cq}}$ method $(33,34)$.

Terminal deoxynucleotidyl-transferase-mediated dUTP nick-end labeling (TUNEL) assay. Apoptosis was determined via a TUNEL assay (One-Step TUNEL Apoptosis assay kit, Beyotime Institute of Biotechnology, Haimen, China) according to the manufacturer's protocols. Briefly, 4- $\mu \mathrm{m}$ thick paraffin sections, which contained the liver tissues were deparaffinized and hydrated, then treated with proteinase 
$\mathrm{K}$ for $20 \mathrm{~min}$ and subsequently incubated with a mixture of fluorescent labeling solution and TdT enzyme at $37^{\circ} \mathrm{C}$ for $1 \mathrm{~h}$ in a humidified atmosphere. The samples were washed in 1XPBS and mounted in mounting media containing DAPI at room temperature for $10 \mathrm{~min}$ without the light. Blue ray was chosen as the exciting light, the wavelength is $420-485 \mathrm{~nm}$. GFP was excited and emitted $515 \mathrm{~nm}$ green fluorescence. Liver cells, which expressed GFP emitted green fluorescent. The total hepatocytes and TUNEL-positive cells were detected in 4-5 randomly selected fields (magnification, x200) for each liver section using a fluorescence microscope (Olympus X71; Olympus Corporation, Tokyo, Japan). The apoptotic rate was calculated according to the formula: Number of TUNEL positive cells/number total cells $x \%(35,36)$. The TUNEL positive cells were calculated by three different authors.

Western blot analysis. Prior to and following reperfusion, all collected liver tissues were rapidly dissected within 5 min and stored at $-80^{\circ} \mathrm{C}$ for cryopreservation. In order to extract the total proteins, the liver tissue was thawed and homogenized in radioimmunoprecipitation assay buffer containing a protease inhibitor (cat. no. G2002; Wuhan Servicebio Co., Ltd., Wuhan, China) and then centrifuged at 20,000 x g for $10 \mathrm{~min}$ at $4^{\circ} \mathrm{C}$. Following collection of the supernatants and measuring the total protein concentration, $30 \mathrm{mg}$ protein, which was calculated by the bicinchoninic acid kit (cat. no. G2026; Wuhan Servicebio Co., Ltd., Wuhan, China) was separated by $10 \%$ SDS-PAGE and transferred to polyvinylidene difluoride membranes (EMD Millipore, Billerica, MA, USA). The membranes were blocked in 5\% skimmed milk for $1 \mathrm{~h}$ at room temperature. The blots were then incubated in $4^{\circ} \mathrm{C}$ for $12 \mathrm{~h}$ with the following antibodies: Rabbit anti-Nrf2 antibody (1:1,000; cat. no. 16396-1-AP; Wuhan Sanying Biotechnology), rabbit anti-HO-1 antibody $(1: 2,000$; cat. no. 27282-1-AP Wuhan Sanying Biotechnology), rabbit anti-NQO1 antibody (1:200; cat. no. bs-23407R Beijing Biosynthesis Biotechnology Co., Ltd., Beijing, China), rabbit anti-Toll-like receptor 4 (TLR4) antibody (cat. no. 19811-1-AP, 1:1,000; Wuhan Sanying Biotechnology), rabbit anti-B-cell lymphoma 2 (Bcl-2) antibody (1:1,000; cat. no. 10435-1-AP; Wuhan Sanying Biotechnology), rabbit anti-Bcl-2-associated X (Bax) antibody (1:1,000; cat. no. 60267-1-Ig; Wuhan Sanying Biotechnology) and anti- $\beta$-actin antibody $(1: 3,000$; ProteinTech Group, Inc., Chicago, IL, USA). Following incubation for $1 \mathrm{~h}$ at room temperature with horseradish peroxidase-conjugated goat anti-rabbit $\operatorname{IgG}(1: 3,000$; cat. no. GB23303; Wuhan Goodbio Technology Co. Ltd., Wuhan, China), the proteins were detected using an enhanced chemiluminescence reagent (cat. no. G2020; Wuhan Servicebio Co., Ltd., Wuhan, China) followed by exposure to X-ray film. Quantification of protein bands was performed using ImageJ v1.42q software (National Institutes of Health, Bethesda, MA, USA).

Statistical analysis. All data were analyzed using SPSS 16.0 statistical software for Windows (SPSS, Inc., Chicago, IL, USA) by one-way analysis of variance with Tukey's post-hoc test. All results are presented as the mean \pm standard deviation ( $n=6$ for each experiment). $\mathrm{P}<0.05$ was considered to indicate a statistically significant difference.
Table I. Rat primer sequences used for reverse transcriptionquantitative-polymerase chain reaction.

\begin{tabular}{cl}
\hline Gene & \multicolumn{1}{c}{ Sequence (5'-3') } \\
\hline NRF2 & \\
F & GAGATATACGCAGGAGAGGG \\
R & CTTTTCAGAAGATGGAGGTTT \\
HO-1 & \\
F & GAAGGCTTTAAGCTGGTGATG \\
R & GGCTGGTGTGTAAGGGATGG \\
NQO1 & \\
F & GGCTGGTTTGAGAGAGTGCT \\
R & ACGTTCATGTCCCCGTGG \\
GST-1 & \\
F & \\
R & CACAGAGACACAGCACAGC \\
GCL & CCTTCCACCTCCAAAACAG \\
F & \\
R & GCAGCTCATTGGTTCATCT \\
$\beta$-actin & TCGTCCCTTCAAAGTCTTT \\
F & \\
R & CCCTGGCTCCTAGCACCAT \\
\hline
\end{tabular}

HO-1, heme oxygenase 1; GCL, glutamate cysteine ligase; GST-1, glutathione-S-transferase-1; NQO1, NAD(P)H:quinine oxidoreductase; NRF2, nuclear factor erythroid 2-related factor 2; F, forward; R, reverse.

\section{Results}

HMP leads to decreased enzymatic levels, reduced intrahepatic resistance and improved liver function compared with the CS group. The activities of ALT and AST enzymes in the perfusate were used to assess the severity of IRI to DCD livers. In the present study, the levels of liver enzymes ALT and AST in the perfusate of the CS group increased significantly compared with in the control group ( $\mathrm{P}<0.01$; Fig. $1 \mathrm{~A}$ and $\mathrm{B}$, respectively). The HMP group exhibited significantly decreased levels of ALT and AST compared with in the CS group ( $\mathrm{P}<0.01$; Fig. $1 \mathrm{~A}$ and $\mathrm{B})$. Additionally, the intrahepatic resistance of livers in the control group was low during reperfusion; however, in the CS group, it was significantly higher compared with in the HMP group at each analyzed time point $(\mathrm{P}<0.01$; Fig. $1 \mathrm{C})$. In addition, bile production in the CS group was significantly lower compared with in the control group ( $\mathrm{P}<0.01$; Fig. 1D). Furthermore, bile production decreased in the HMP group; however, no significant difference was observed compared with in the CS group $(\mathrm{P}>0.05)$. Visual and numerical analyses of the histological images indicated that there were few observable abnormalities in the livers of the control group (Fig. 2). Conversely, in the CS group, histological analysis revealed significant congestion of the hepatic sinusoid, vacuolar degeneration and necrosis $(\mathrm{P}<0.01$; Fig. 2A-C). Additionally, significantly attenuated liver damage was observed in the HMP group compared with in the CS group $(\mathrm{P}<0.05$; Fig. 2A-C). 
A

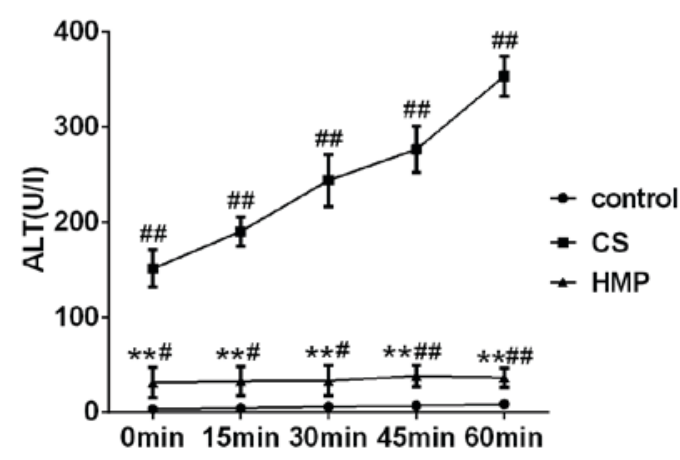

C

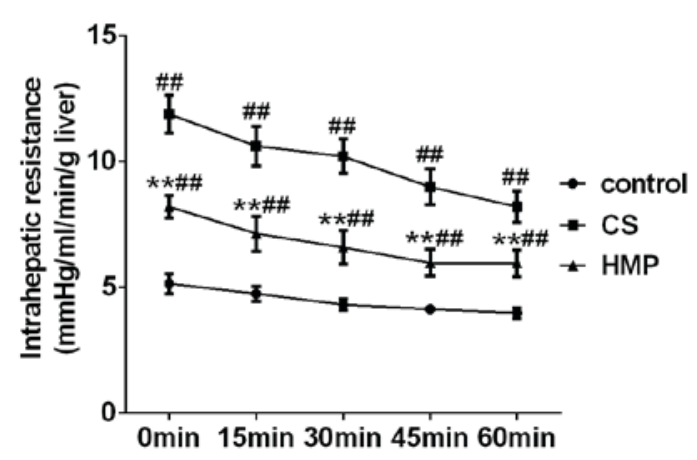

B

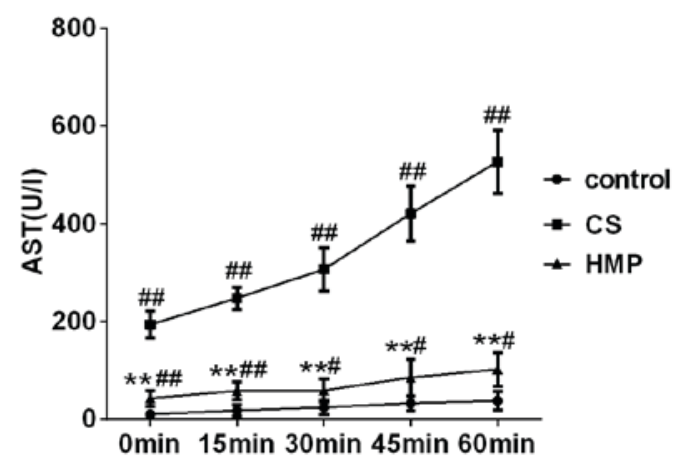

D

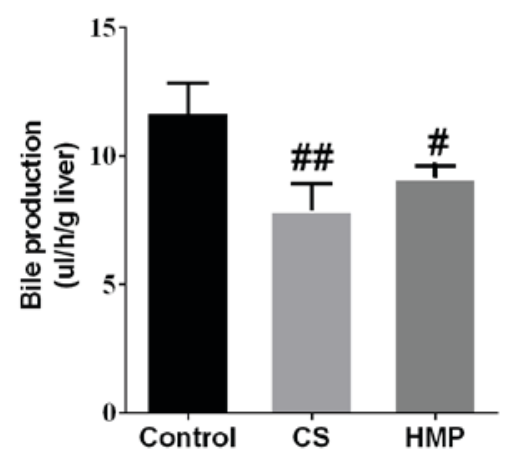

Figure 1. Liver enzyme release, intrahepatic resistance and bile production during reperfusion. During reperfusion, intrahepatic resistance and bile production was continuously monitored. The perfusate was collected to measure liver enzyme release. Levels of (A) ALT and (B) AST in each group. (C) Intrahepatic resistance and (D) bile production in each group. ${ }^{\#} \mathrm{P}<0.05,{ }^{\# \#} \mathrm{P}<0.01$ vs. the control group. ${ }^{* *} \mathrm{P}<0.01$ vs. the CS group. (n=6). ALT, alanine transaminase AST, aspartate amino transferase; CS, cold storage; HMP, hypothermic machine perfusion.

HMP reduces hepatic oxidative stress, inflammation and apoptosis. The present study analyzed the typical biochemical markers of oxidative stress to further investigate the effects of HMP on the IRI livers. Oxidative stress has been considered to be an important factor leading to IRI. The SOD activities and expression levels of MDA of the tissues were presented in Fig. 3A and B. Compared with the control group, SOD activity in the CS group was significantly decreased $(\mathrm{P}<0.01)$, and the expression levels of MDA were significantly increased $(\mathrm{P}<0.01)$. The results of the present study indicated that HMP treatment may significantly decrease the MDA expression levels $(\mathrm{P}<0.01)$ and significantly increase the SOD activity $(\mathrm{P}<0.01)$; opposite trends to those observed in the CS group. These results suggested that HMP may attenuate oxidative stress. In addition, the ATP content in the liver was also measured to analyze liver function. As presented in Fig. 3C, within the CS group, the ATP content was significantly lower compared with the control group $(\mathrm{P}<0.01)$; however, HMP treatment significantly increased the ATP content compared with in the CS group. $(\mathrm{P}<0.01)$.

Oxidative stress can also induce hepatic inflammation and apoptosis $(37,38)$. Therefore, the present study investigated the expression levels apoptosis-associated proteins in the liver (Fig. 3D). The ratio of Bcl-2 to Bax is also important in determining susceptibility of cells to apoptosis (39). The overexpression of Bax revealed that apoptosis was promoted in response to a death-inducing signal, which suggested its role as an apoptosis agonist (40). Bcl-2 overexpression has been associated with heterodimerization with Bax and reduced levels of apoptosis (41). In addition, TLR4 has been reported to be an important marker of inflammation (42). As presented in Fig. 3E, the ratio of Bcl-2 to Bax was significantly downregulated in the CS group and significantly upregulated in the HMP group $(\mathrm{P}<0.01)$. The protein expression levels of TLR4 were significantly increased in the CS group and significantly decreased in the HMP group $(\mathrm{P}<0.01$; Fig. 3F). Furthermore, the present study revealed that, compared with the control group, the rate of apoptosis in the CS group was significantly increased ( $\mathrm{P}<0.01$; Fig. 4); however, treatment with HMP was associated with significantly reduced rates of apoptosis.

HMP activates the Keapl/Nrf2-ARE antioxidant pathway in DCD rat livers. Nrf2 serves an important role in the main defense mechanisms induced by cellular oxidative stress $(10,11)$. Therefore, the present study investigated whether HMP conferred protection against IRI to rat DCD livers via alterations in Nrf2 expression. The results reveled that compared with the control group, the expression levels of Nrf2 in the CS group were significantly decreased $(\mathrm{P}<0.05)$; however, the HMP group exhibited a significant increase compared with in the CS group $(\mathrm{P}<0.01$; Fig. 5A and $\mathrm{B})$. In addition to the expression levels of Nrf2 protein, NQO1 protein 
A

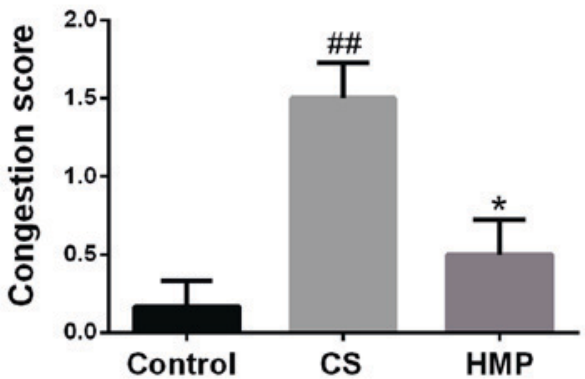

C

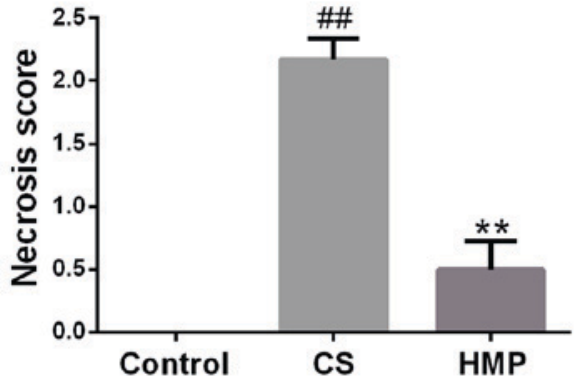

CS

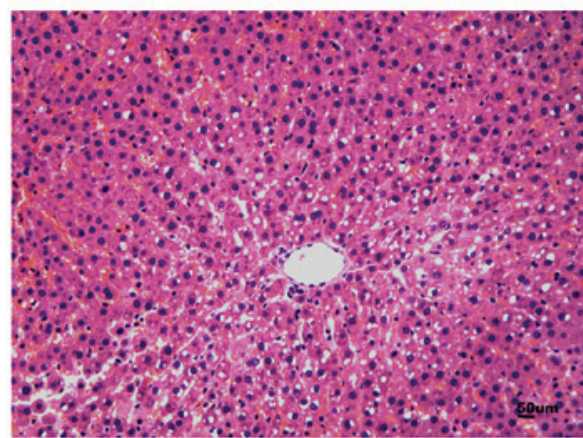

B

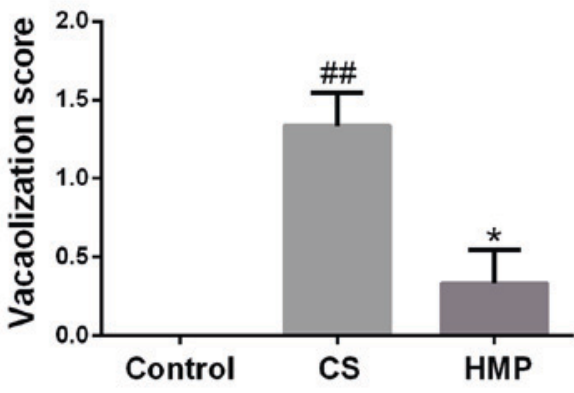

Control

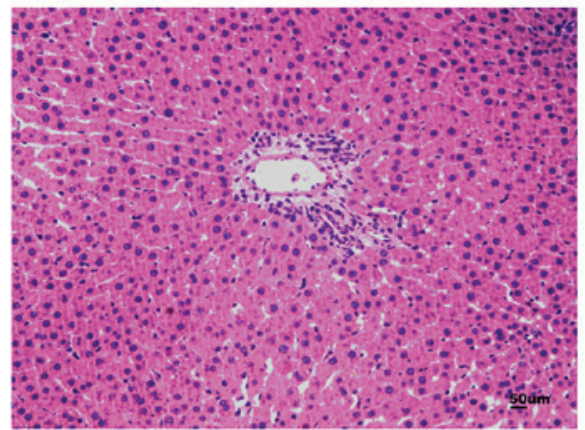

HMP

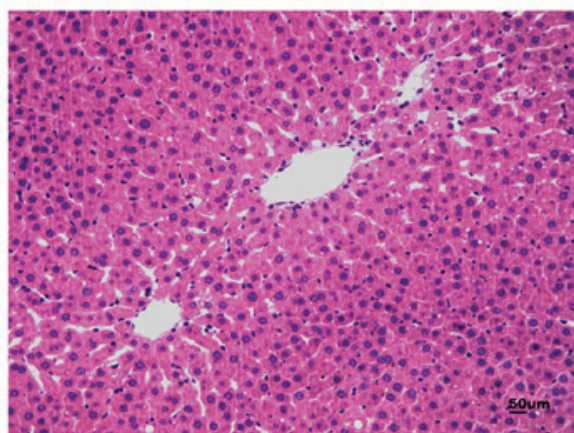

Figure 2. Liver histopathology. Extent of histological damage in the control, CS and HMP groups were quantified according to (A) congestion, (B) vacuolization and $(\mathrm{C})$ necrosis score via (D) histological observation. Compared with the control and HMP groups, the CS group exhibited severe liver injury. ${ }^{\# \prime} \mathrm{P}<0.01$ vs. the control group. HMP significantly reduced the degree of injury compared with in the CS group. ${ }^{*} \mathrm{P}<0.05,{ }^{* *} \mathrm{P}<0.01$ vs. the CS group. Magnification, $\mathrm{x} 200$. CS, cold storage; HMP, hypothermic machine perfusion

expression levels in the CS and HMP groups demonstrated opposing expression patterns ( $\mathrm{P}<0.05$; Fig. 5A and B). Notably, despite the significant increase in $\mathrm{HO}-1$ protein expression levels in the CS group compared with the control, the HMP group exhibited a significant decrease compared with the CS group $(\mathrm{P}<0.01$; Fig. 5A and $\mathrm{B})$.

Reperfusion injury due to toxic reactive oxygen species generated upon reintroduction of blood flow and oxygen supply to ischemic tissues is the main cause of DCD liver injury (43). Therefore, the expression levels of proteins in the presence or absence of reperfusion were investigated in the present study to determine whether HMP may activate the Nrf2-ARE signaling pathway.

The results of the present study revealed that the expression levels of Nrf2, NQO-1 and HO-1 were all been significantly upregulated during reperfusion in both the CS and HMP groups $(\mathrm{P}<0.05$; Fig. 5C and $\mathrm{D})$. In addition, the induction of Nrf2 $(0.392 \pm 0.137$ vs. $0.912 \pm 0.122 ; \mathrm{P}<0.0001)$ and NQO-1 $(0.342 \pm 0.057$ vs. $1.076 \pm 0.102 ; \mathrm{P}<0.0001)$ expression in the presence of IRI was significantly higher within the HMP group compared with the induction of $\mathrm{Nrf} 2(0.300 \pm 0.079$ vs. $0.529 \pm 0.075 ; \mathrm{P}=0.007)$ and $\mathrm{NQO}-1(0.287 \pm 0.104$ vs. $0.575 \pm 0.078 ; \mathrm{P}<0.0001)$ expression in the CS group. All this suggested that the effects of HMP on oxidative stress may occur via activation of the Nrf2-ARE signaling pathway $(\mathrm{P}<0.05$; Fig. 5C and D).

Additionally, the mRNA expression levels of Nrf2, HO-1, NQO1, GST-1, and GCL in the liver were investigated via RT-qPCR (Fig. 6). The results of the present study revealed that except for HO-1, the mRNA expression levels of the other ARE-regulated genes were significantly enhanced in the HMP group compared with the CS group $(\mathrm{P}<0.05$; Fig. 6). This suggested that additional regulatory elements may be involved in the activation of HO-1 in the CS group; however, the activation of Nrf2 and other ARE-regulated genes, as well as the inhibition of oxidative stress markers, including MDA in the HMP group, suggested that HMP may be responsible for the increase in antioxidative ability of cells by activating the Nrf2-ARE signaling pathway.

Furthermore, previous studies have confirmed that Nrf2 may be activated in endothelial cells by steady laminar flow $(19,20)$. Thus, immunohistochemistry was conducted to assess whether 
A

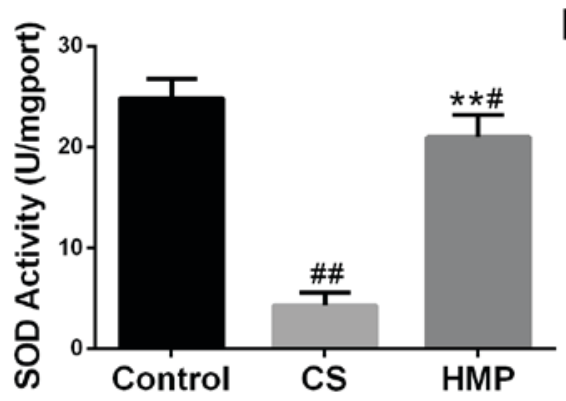

C

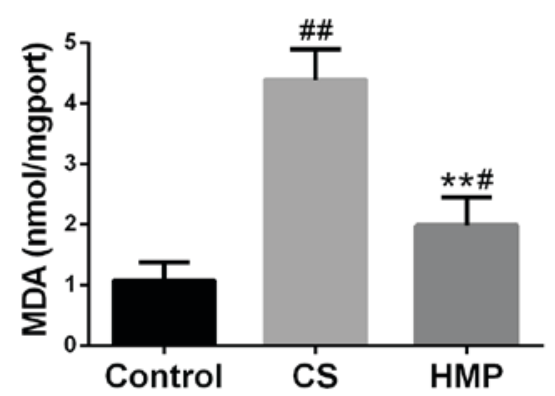

$B$

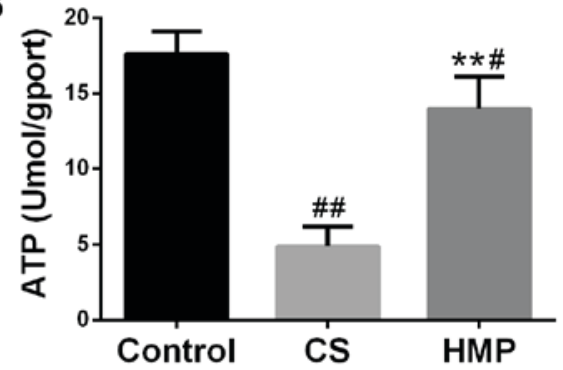

D

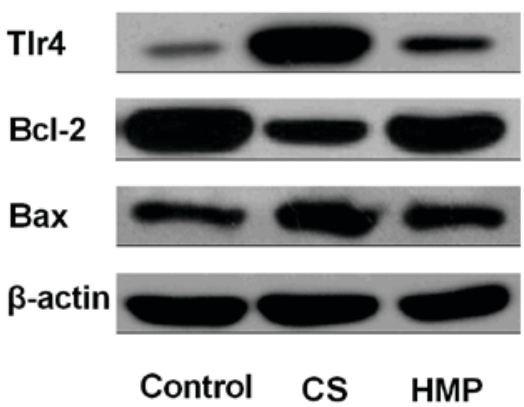

E

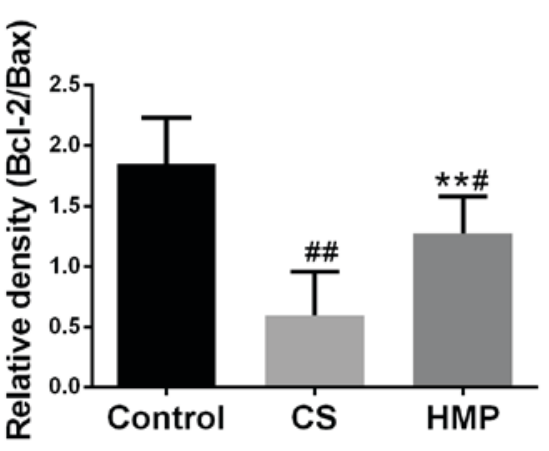

\section{$F$}

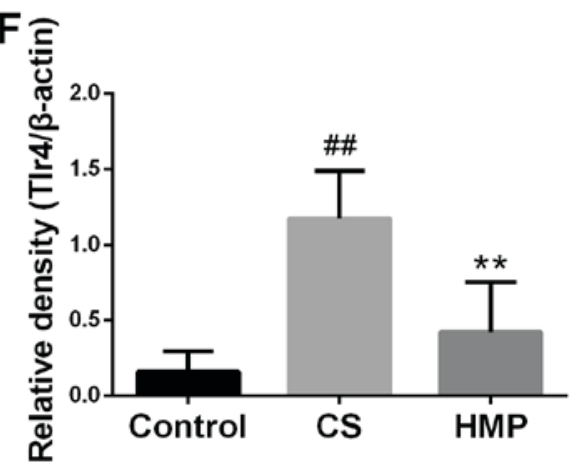

Figure 3. Effects of HMP on the index of inflammation and apoptosis. Following reperfusion, (A) SOD activity, (B) MDA expression levels and (C) ATP content in the liver tissues of each group were assessed using respective commercial kits. (D) Protein expression levels of Tlr4, Bcl-2 and Bax in the livers of each group were analyzed by western blotting. $\beta$-actin served as an internal control. Compared with in the CS group, the (E) Bcl-2/Bax ratio was significantly increased; however, the expression levels of (F) TLR4 protein were decreased. ${ }^{\#} \mathrm{P}<0.05,{ }^{\# \#} \mathrm{P}<0.01$ vs. the control group; ${ }^{* *} \mathrm{P}<0.01 \mathrm{vs}$. the CS group. Bcl-2, B-cell lymphoma-2; Bax, Bcl-2 associated X; CS, cold storage; DCD, donated after cardiac death; HMP, hypothermic machine perfusion; MDA, malondialdehyde; SOD, superoxide dismutase; TLR4, Toll like receptor 4.

steady laminar flow provided by HMP may induce the expression levels of Nrf2 (Fig. 7). The results revealed that within the HMP group, Nrf2 expression was significantly increased in the periportal regions of livers when compared with the CS group $(\mathrm{P}<0.01)$, This suggested that Nrf2 in hepatocytes and liver sinusoidal endothelial cells around periportal regions may be induced by steady laminar flow.

\section{Discussion}

HMP has become a topic of interest in the past decade, with regards to maintaining the quality of liver grafts, particularly DCD livers (44); however, the protective mechanisms of HMP require further investigation. The present study investigated the benefits of HMP in mitigating injuries from preservation methods with a specific focus on oxidative stress in DCD livers. The Nrf2 signaling pathway is an important mediator of the antioxidant system in mammals (15). In the present study, it was demonstrated that HMP may reduce IRI to DCD livers via activation of the Nrf2-ARE signaling pathway.

Additionally, the present study demonstrated that, compared with the CS group, the HMP group revealed improved hepatocellular functions and overall tissue viability, as indicated by improved ATP and bile production, and lower ALT and AST levels. Bile production between the CS group and HMP group did not exhibit significant differences under the HMP condition, and this may have been due to the delivery of nutrients and oxygen via the portal vein rather than the hepatic artery, which is the only way oxygen is delivered in the physiological state $(23,31)$. In HMP group the liver was perfused through 


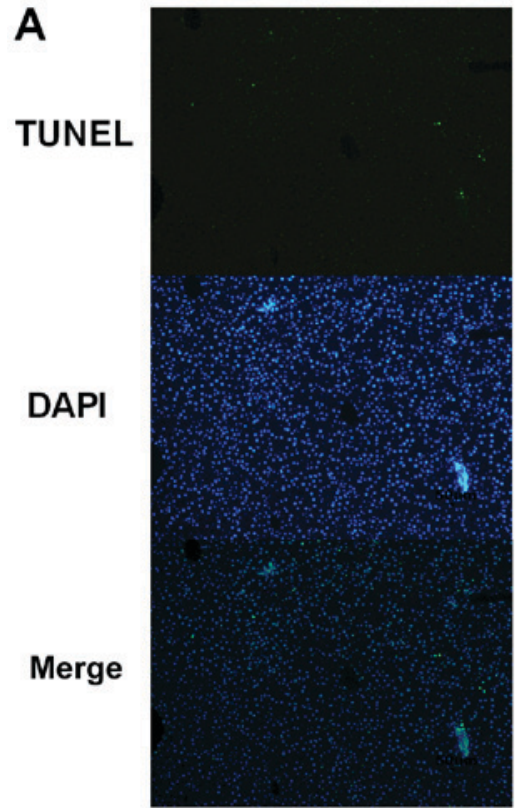

Control

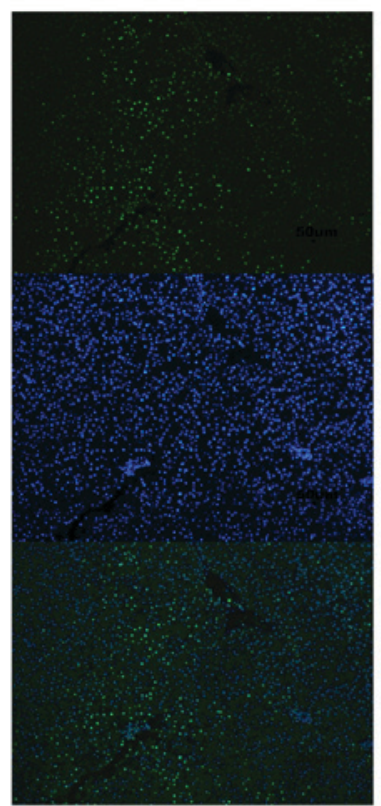

CS

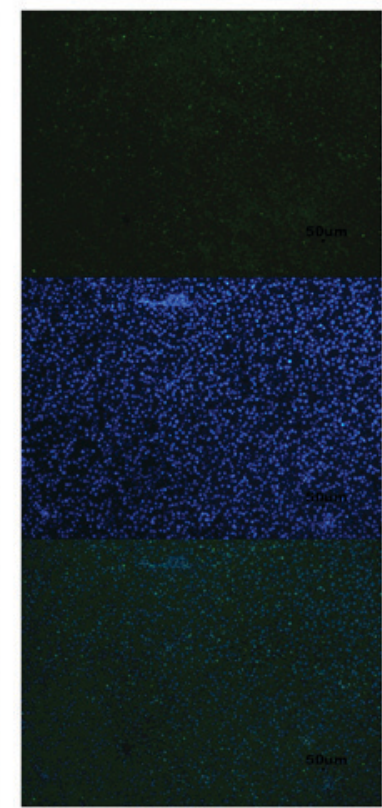

HMP

B

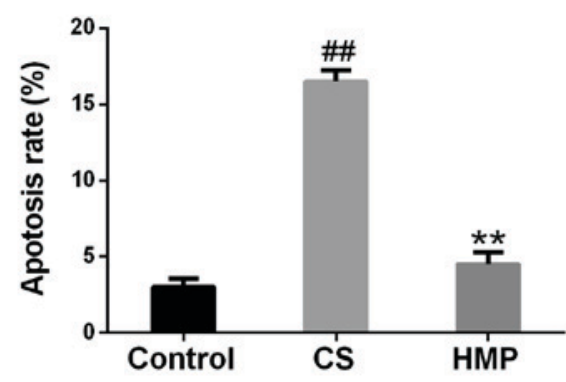

Figure 4. Apoptosis of rat liver following reperfusion as determined by TUNEL assay. (A) The nuclei of liver cells were stained with 4',6-diamidino-2-phenylindole and apoptosis was assessed by TUNEL staining. The percentage of apoptotic cells was determined by counting 4-5 microscopic fields per liver. Magnification, $\mathrm{x} 200$. (B) Quantitative analysis of apoptosis. ${ }^{\# \prime} \mathrm{P}<0.05$, vs. the control group; ${ }^{* *} \mathrm{P}<0.01$, vs. the CS group. $(\mathrm{n}=6)$. CS, cold storage; HMP, hypothermic machine pressure; TUNEL, terminal deoxynucleotidyl-tranferase-mediated nick-end labeling.

portal vein, therefore cholangiocytes may be more sensitive to ischemic injury and would influence the production of bile. The congestion and vacuolar degeneration observed in the histological sections were considered to be possible causes for microcirculation dysfunction. In addition to the improved histological findings, the levels of intrahepatic resistance in the HMP group were significantly lower compared with in the CS group.

Numerous mechanisms underlying IRI in the DCD liver grafts have been reported, including oxidative stress (45). In the present study, the levels of SOD activity represented the antioxidant capacity and MDA expression levels reflected the degree of oxidative damage to cells. The present study observed a significant reduction in the levels of MDA within the HMP group, indicating that oxidative damage to the graft may be attenuated following HMP preservation compared with in the CS group. Furthermore, an increase in the activity of SOD following reperfusion was observed during HMP preservation, which indicated a cellular attempt to ameliorate oxidative damage. Overall, the findings of the present study suggested that HMP may be a better strategy of preservation than CS and may exert protective effects against IRI on the DCD liver.
Oxidative stress may also induce hepatic inflammation and apoptosis (37-39). In addition, TLR4 is an important marker of inflammation and is activated during hepatic IR, as well as IRI in multiple organs; the importance of TLR4 in IRI has also been verified in a transplant model (46). The results of the present study indicated that the protein expression levels of TLR4 in the CS group were significantly higher than the control group; however, the HMP group exhibited lower expression levels of TLR4 than the CS group. This result suggested that reductions in oxidative stress may reduce the inflammation of DCD liver. In addition, Bax and Bcl-2 are important markers of apoptosis (40); $\mathrm{Bax}$ is a proapoptotic protein, while $\mathrm{Bcl}-2$ is an antiapoptotic protein (41). Thus, the protein expression levels of Bcl-2 and Bax following reperfusion were investigated in the present study to determine whether apoptosis may be inhibited. The alterations of the Bcl-2/Bax ratio in the CS and HMP groups indicated that HMP may attenuate hepatocyte apoptosis. TUNEL staining also indicated the extent of hepatocyte apoptosis in the present study. When investigations were performed with HMP instead of CS to preserve the liver, the rate of hepatocyte apoptosis decreased significantly in the present study. This indicated that, with the decline of liver oxidative stress, liver inflammation and apoptosis may be attenuated. 
A

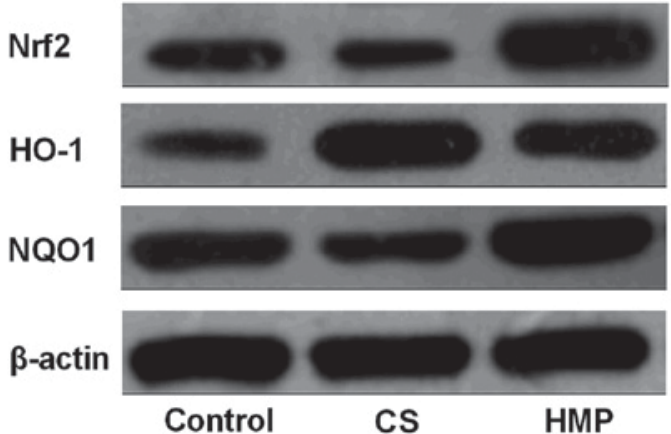

C

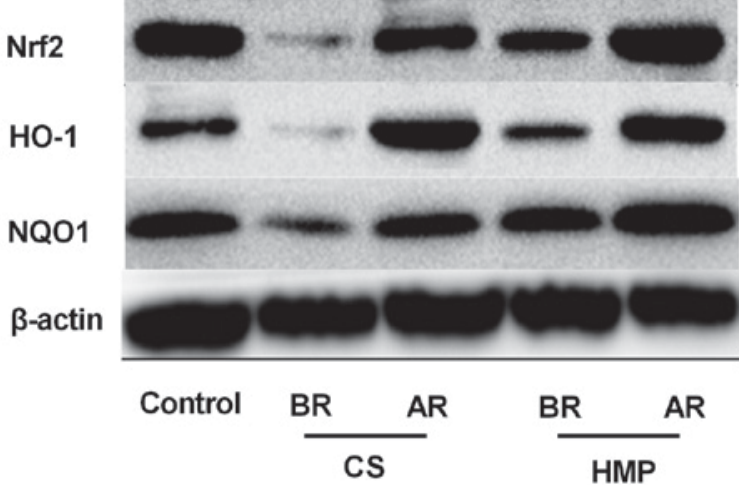

B

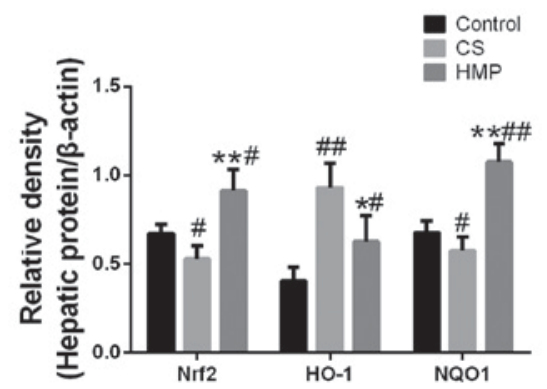

D

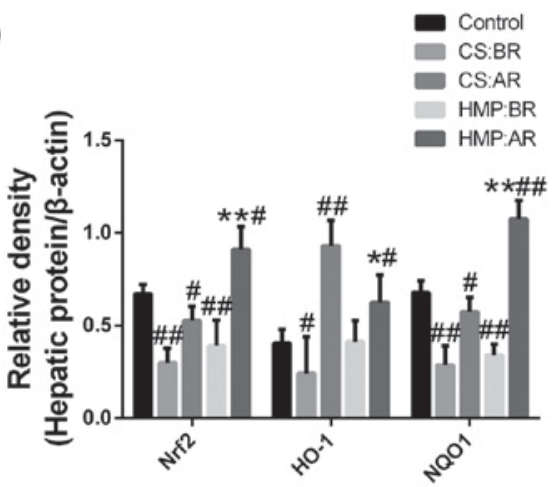

Figure 5. Effect of HMP on Nrf2, HO-1 and NQO1 protein expression levels in donated after cardiac death rat liver models. (A and B) Following reperfusion, the protein expression levels of Nrf2, HO-1 and NQO1 in the livers of the control, CS and HMP groups were analyzed by western blotting. $\beta$-actin served as an internal control. ${ }^{\#} \mathrm{P}<0.05,{ }^{\# \#} \mathrm{P}<0.01$ vs. the control group, ${ }^{*} \mathrm{P}<0.05,{ }^{* *} \mathrm{P}<0.01$ vs. the CS group. (C and D) Western blotting and the quantitative analysis revealed the expression levels of the components associated with the Nrf2-ARE pathway in the livers of the CS and HMP groups prior to and following reperfusion. ${ }^{\#} \mathrm{P}<0.05,{ }^{\# \#} \mathrm{P}<0.01$, the CS group vs. the BR CS group; and HMP group vs. the BR HMP group, respectively. AR, after reperfusion; BR, before reperfusion; CS, cold storage; HMP, hypothermic machine pressure; HO-1, heme oxygenase-1; NQO1, NAD(P)H:quinine oxidoreductase 1; Nrf2, nuclear factor erythroid 2-related factor 2 .

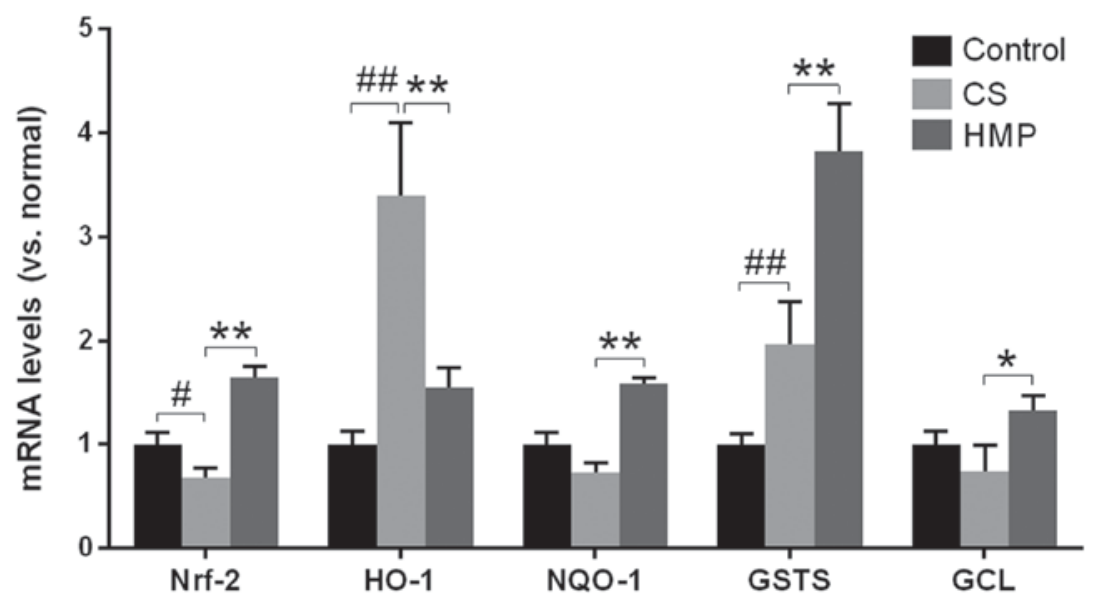

Figure 6. Fold alterations in Nrf-2, HO-1, NQO1, GST-1, and GCL mRNA expression levels following reperfusion of rat livers. Reverse transcription-quantitative polymerase chain reaction revealed that the expression of antioxidant response element-containing genes (HO-1, NQO1, GST-1 and GCL) exhibited significant alterations between the CS and HMP groups. The quantification cycle values were quantified by the ratio of target relative to housekeeping gene $\beta$-actin and the differences were calculated by the $2^{-\Delta \Delta C q}$ method. ${ }^{\#} \mathrm{P}<0.05,{ }^{\# \#} \mathrm{P}<0.01$ vs. the control group; ${ }^{*} \mathrm{P}<0.05,{ }^{* *} \mathrm{P}<0.01$ vs. the CS group. CS, cold storage; GCL, glutamate cysteine ligase; GST-1, glutathione-S-transferase-1; HO-1, heme oxygenase-1; HMP, hypothermic machine pressure; NQO1, NADPH NAD(P)H:quinine oxidoreductase 1; Nrf2, nuclear factor erythroid 2-related factor 2.

Previous studies have suggested a role of Nrf2 in antioxidative and anti-apoptotic process functions in IRI $(16,47)$. Therefore, the present study proposed that the activity of Nrf2 may serve a role in the protective effects of HMP against IRI; the protein and mRNA expression levels of Nrf2 between the
HMP and CS groups were investigated. Significantly higher expression levels were observed in the HMP group compared with in the CS, which indicated that HMP may have induced the expression of Nrf2. Additionally, immunohistochemistry analysis demonstrated that the expression of Nrf2 in the 
A

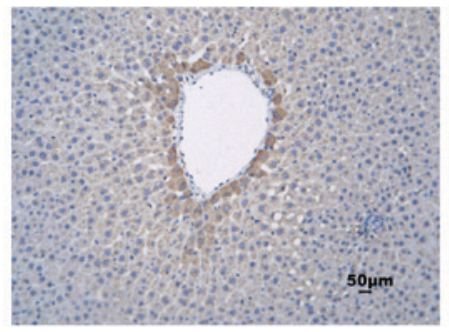

Control

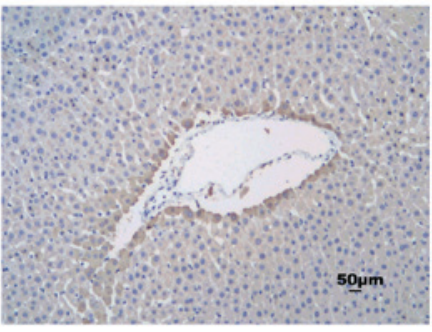

cs

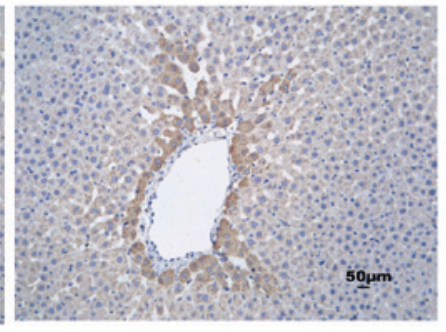

HMP

B

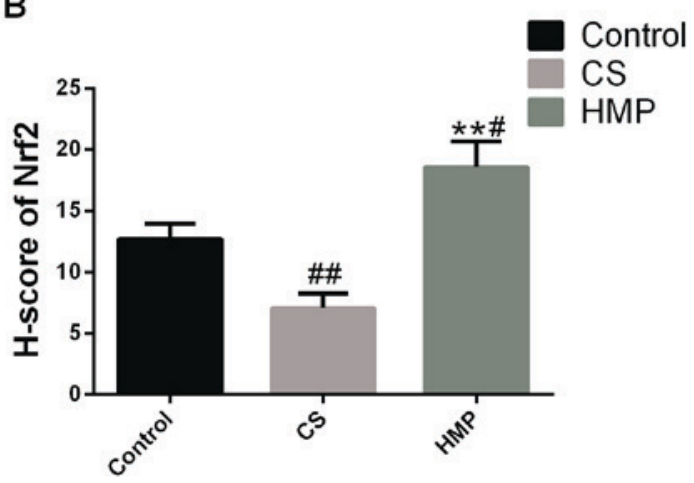

Figure 7. Immunohistochemical localization of Nrf2 in the liver following reperfusion. (A) The pericentral region, which was stained with an antibody against Nrf2 in the control, CS and HMP groups. Scale bar=50 $\mu \mathrm{m}$. Magnification, $\mathrm{x} 200$. (B) The histochemical score of each group. ${ }^{\text {} P} \mathrm{P}<0.05$, ${ }^{\# \prime} \mathrm{P}<0.05$, compared with the control group; ${ }^{* *} \mathrm{P}<0.01$, compared with the CS group. CS, cold storage; HMP, hypothermic machine pressure; Nrf2, nuclear factor erythroid 2-related factor 2 .

pericentral region was notably increased following HMP treatment. The Nrf2 signaling pathway mainly involves Nrf2, Keap1 and ARE. Following dissociation from Keap1, activated Nrf2 enters the nucleus and binds to the ARE sequence by combining with MAF protein to form heterodimers. Through this binding process, Nrf2 regulates the transcription of antioxidative-associated genes (15). Following reperfusion, the expression of antioxidant molecules, including GST-1, NQO1 and GCL, which are the target genes of Nrf2, are increased in the livers of the HMP group. As described in the previous results, compared with the control group, the expression levels of HO-1 protein appeared to increase more than in the HMP group. The reason for this phenomenon may be that the regulation of HO-1 gene expression occurs at the transcriptional level. The regulatory elements of the gene include not only components associated with the Nrf2-ARE signaling pathway, but also the activator protein-1 binding site, nuclear factor- $\kappa \mathrm{B}$ binding site, a heat-shock element and hypoxic response elements. Activation of the HO-1 gene results from the binding of various transcription factors to these regulatory elements (48-50). There may be other potential mechanisms underlying the increased protein expression levels of HO-1 in the CS group.

Providing that reperfusion is the main cause of DCD liver injury, the present study also investigated the expression of proteins associated with the Nrf2-ARE signaling pathway prior to and following reperfusion to determine whether HMP may activate this signaling pathway. The protein expression levels of the Nrf2-ARE signaling pathway were significantly different prior to and following reperfusion. In addition, the higher end protein level quantification values of Nrf2 and NQO1 in the HMP group compared with the CS group following reperfusion indicated that the effect of HMP was more notable than CS treatment. In addition, the present study revealed that HMP may be able to reduce the extent of apoptosis and inflammation in accordance with alterations in Nrf2 expression. This indicated that the Nrf2-ARE signaling pathway may serve an important role in the molecular mechanism underlying the protective effects of HMP.

Furthermore, previous studies have confirmed that Nrf2 may be activated by steady laminar flow $(19,20,51)$. When endothelial cells come into contact with the flowing blood, they experience different types of stress. In contrast with disturbed blood flow, steady laminar flow serves positive roles, and in this situation the activation of protective factors including Nrf2 is dominant (20). Based on previous studies, steady laminar flow leads to sustained high shear stress, and flow shear stress were considered to produce antioxidant, antiapoptotic, anti-inflammatory, and antiproliferative effects $(20,51)$. Therefore, the results of the present study that HMP demonstrated decreased enzymatic levels, reduced intrahepatic resistance and improved histological findings compared with CS, suggest that steady laminar flow may provides a supply of metabolic substrates and removes byproducts, recreating the normal circulation, which corresponds with the previous studies (52). Then, Hsieh et al (51) demonstrated that shear stress can increase Nrf2 protein expression and induce Nrf2 translocation into nuclei; in addition, shear stress also increased the ARE-binding activity of Nrf2, and a number of antioxidant genes, including HO-1, NQO1 and GST-1, are upregulated in endothelial cells under laminar shear stress. 
The activation of Nrf2 is essential for the antioxidant function of shear stress, and the effector proteins HO-1 and NQO1 have been demonstrated to respond to flow shear stress in vascular endothelial cells (19). However the detailed mechanism of the activation of Nrf 2 and how the antioxidant ability of flow shear stress is regulated remains to be studied. Additionally, as this perspective suggests, immunohistochemistry analysis in our study also demonstrated that the expression of Nrf2 which may be activated in endothelial cells by steady laminar flow, was notably increased in the pericentral region following HMP treatment. As a result of all this information, HMP preservation was concluded to dilate the intrahepatic vasculature and improve microcirculation, resulting in steady laminar flow during reperfusion, which was the activator of Nrf2-ARE pathway.

In the present study, the alterations of NRF2-ARE pathway by different storage method during simulated DCD liver transplantation were investigated. NRF2 is a key molecule in anti-oxidative stress $(10,11)$. Combined with the authors' studies, this may suggest that the NRF2-ARE signaling pathway may be a novel pharmacological target for improving DCD liver quality. However, the present study also has some deficiencies. Due to the lack of a murine IPRL system, the present study failed to use knockout mice, which may need further study. Organ preservation has always been a bottleneck that affected the development of transplantation. How to preserve organs more effectively has always been a study hotspot in the field of transplantation. In addition, although HMP is the routine method for clinical donated kidney protection, which kind of preservation method is more effective in the liver remains unknown. It was demonstrated that the possible mechanism of HMP to protect the DCD liver maybe result from the activation of NRF2-ARE pathway, but the specific mechanism needs to be further research by using the knockout mice. In addition, clinical trials may focus on drugs associated with NRF2 pathway to improve DCD liver and the outcome of the transplant. These are possible future research areas. In conclusion, the protective effects of HMP on the DCD liver may arise from alterations in Nrf2 expression levels. Therefore, via the activation of Nrf2 and its function of binding to the ARE sequence, HMP may serve a role against oxidative stress to protect the DCD liver from IRI.

\section{Acknowledgements}

The authors would like to thank the professors and students from Zhongnan Hospital of Wuhan University and Institute of Hepatobiliary Diseases of Wuhan University, who participated in this study.

\section{Funding}

The present study was supported by the National Natural Science Foundation of China (grant no. U1403222).

\section{Availability of data and materials}

All data generated or analyzed during this study are included in this published article.

\section{Authors' contributions}

SX and QY contributed the central idea, analyzed most of the data, and wrote the initial draft of the paper. WH, XZ, ZZ, YX and YW contributed to refining the ideas. SX, QY, WH, XZ, $\mathrm{ZT}$ and SF carried out additional analyses and finalizing this paper.

\section{Ethics approval and consent to participate}

The present study was conducted according to the Experimental Animal Regulations of the People's Republic of China and the Guide for the Care and Use of Laboratory Animals of the National Institutes of Health, ensuring that all animals received humane care. The present study was approved by the Ethics Committee of Wuhan University (Wuhan, China).

\section{Consent for publication}

Not applicable.

\section{Competing interests}

The authors declare that they have no competing interests.

\section{References}

1. O'Leary JG, Lepe R and Davis GL: Indications for liver transplantation. Gastroenterology 134: 1764-1776, 2008.

2. Monbaliu D, Pirenne J and Talbot D: Liver transplantation using donation after cardiac death donors. J Hepatol 56: 474-485, 2012.

3. Neyrinck A, Van Raemdonck D and Monbaliu D: Donation after circulatory death: Current status. Curr Opin Anaesthesiol 26: 382-390, 2013.

4. Jay CL, Lyuksemburg V, Ladner DP, Wang E, Caicedo JC, Holl JL, Abecassis MM and Skaro AI: Ischemic cholangiopathy after controlled donation after cardiac death liver transplantation: A meta-analysis. Ann Surg 253: 259-264, 2011.

5. Fujita S, Mizuno S, Fujikawa T, Reed AI, Kim RD, Howard RJ and Hemming AW: Liver transplantation from donation after cardiac death: A single center experience. Transplantation 84: 46-49, 2007.

6. Manzarbeitia CY, Ortiz JA, Jeon H, Rothstein KD, Martinez O, Araya VR, Munoz SJ and Reich DJ: Long-term outcome of controlled, non-heart-beating donor liver transplantation. Transplantation 78: 211-215, 2004.

7. Morrissey PE and Monaco AP: Donation after circulatory death: Current practices, ongoing challenges, and potential improvements. Transplantation 97: 258-264, 2014

8. Henry SD and Guarrera JV: Protective effects of hypothermic ex vivo perfusion on ischemia/reperfusion injury and transplant outcomes. Transplantation Rev (Orlando) 26: 163-175, 2012.

9. Schreinemachers MJ, Doorschodt BM and van Gulik TM: Machine perfusion preservation of the liver: A worthwhile clinical activity? Curr Opin Organ Transplant 12: 224-230, 2007.

10. Ma Q and He X: Molecular Basis of Electrophilic and Oxidative Defense: Promises and Perils of Nrf2. Pharmacol Rev 64: 1055-1081, 2012.

11. Kensler TW, Wakabayashi N and Biswal S: Cell survival responses to environmental stresses via the Keap1-Nrf2-ARE pathway. Annu Rev Pharmacol Toxicol 47: 89-116, 2007.

12. Itoh K, Wakabayashi N, Katoh Y, Ishii T, Igarashi K, Engel JD and Yamamoto M: Keap1 represses nuclear activation of antioxidant responsive elements by Nrf2 through binding to the amino-terminal Neh2 domain. Genes Dev 13: 76-86, 1999.

13. Kang MI, Kobayashi A, Wakabayashi N, Kim SG and Yamamoto M: Scaffolding of Keap1 to the actin cytoskeleton controls the function of Nrf2 as key regulator of cytoprotective phase 2 genes. Proc Natl Acad Sci USA 101: 2046-2051, 2004 . 
14. Zhang DD and Hannink M: Distinct cysteine residues in keap1 are required for Keap1-dependent ubiquitination of Nrf2 and for stabilization of $\mathrm{Nrf} 2$ by chemopreventiveagents and oxidative stress. Mol Cell Biol 23: 8137-8151, 2003.

15. Kurokawa H, Motohashi H, Sueno S, Kimura M, Takagawa H, Kanno Y, Yamamoto M and Tanaka T: Structural basis of alternative DNA recognition by Maf transcription factors. Mol Cell Biol 29: 6232-6244, 2009.

16. Kudoh K, Uchinami H, Yoshioka M, Seki E and Yamamoto Y: $\mathrm{Nrf} 2$ activation protects the liver from ischemia/reperfusion injury in mice. Ann Surg 260: 118-127, 2014.

17. Lee LY, Harberg C, Matkowskyj KA, Cook S, Roenneburg D, Werner S, Johnson J and Foley DP: Overactivation of the nuclear factor (erythroid-derived 2)-like 2-antioxidant response element pathway in hepatocytes decreases hepatic ischemia/reperfusion injury in mice. Liver Transplant 22: 91-102, 2016.

18. Tanaka Y, Maher JM, Chen C and Klaassen CD: Hepatic ischemia-reperfusion induces renal heme oxygenase-1 via NF-E2-related factor 2 in rats and mice. Mol Pharmacol 71: 817-825, 2007.

19. Fukuda Y, Kaishima M, Ohnishi T, Tohyama K, Chisaki I, Nakayama Y, Ogasawara-Shimizu M and Kawamata Y: Fluid shear stress stimulates MATE2-K expression via Nrf2 pathway activation. Biochem Biophys Res Commun 484: 358-364, 2017.

20. Nigro P, Abe J and Berk BC: Flow shear stress and atherosclerosis: A matter of site specificity. Antioxid Redox Signal 15: 1405-1414, 2011.

21. Zakkar M, Angelini GD and Emanueli C: Regulation of vascular endothelium inflammatory signalling by shear stress. Curr Vasc Pharmacol 14: 181-186, 2016.

22. National Research Council: Guide for the care and use of laboratory animals. 8th edition. The National Academies press, Washington, DC, 2011

23. Carnevale ME, Balaban CL, Guibert EE, Bottai $\mathrm{H}$ and Rodriguez JV: Hypothermic machine perfusion versus cold storage in the rescuing of livers from non-heart-beating donor rats. Artif Organs 37: 985-991, 2013.

24. Rodriguez J, Guibert EE, Quintana A, Scandizzi A and Almada L: Role of sodium nitroprusside in the improvement of rat liver preservation in University of Wisconsin solution: A study in the isolated perfused liver model. J Surg Res 87: 201-208, 1999.

25. Dutkowski P, Schönfeld S, Odermatt B, Heinrich T and Junginger T: Rat liver preservation by hypothermic oscillating liver perfusion compared to simple cold storage. Cryobiology 36: 61-70, 1998.

26. Zeng $\mathrm{C}, \mathrm{Hu} \mathrm{X}, \mathrm{He} \mathrm{W}$, Wang Y, Li L, Xiong Y and Ye Q: Hypothermic machine perfusion ameliorates inflammation during ischemia-reperfusion injury via sirtuin-1-mediated deacetylation of nuclear factor- $\kappa \mathrm{B}$ p65 in rat livers donated after circulatory death. Mol Med Rep 16: 8649-8656, 2017.

27. Schlegel A, Kron P, Graf R, Dutkowski P and Clavien PA: Warm vs. cold perfusion techniques to rescue rodent liver grafts. J Hepatol 61: 1267-1275, 2014.

28. Pizarro MD, Rodriguez JV, Mamprin ME, Fuller BJ, Mann BE, Motterlini R and Guibert EE: Protective effects of a carbon monoxide-releasing molecule (CORM-3) during hepatic cold preservation. Cryobiology 58: 248-255, 2009.

29. Srinivasan PK, Yagi S, Doorschodt B, Nagai K, Afify M, Uemoto $S$ and Tolba R: Impact ofvenous systemic oxygen persufflation supplemented with nitric oxide gas on cold-stored, warm ischemia-damaged experimental liver grafts. Liver Transpl 18: 219-225, 2012.

30. Minor T and Manekeller S: Assessment of hepatic integrity after ischemic preservation by isolated perfusion in vitro: The role of albumin. Cryobiology 54: 188-195, 2007.

31. Boyer JL: Bile formation and secretion. Compr Physiol 3: 1035-1078, 2013

32. Suzuki S, Toledo-Pereyra LH, Rodriguez FJ and Cejalvo D: Neutrophil Infiltration As An Important Factor in Liver Ischemia and Reperfusion Injury. Transplantation 55: 1265-1272, 1993.

33. Funel N, Giovannetti E, Chiaro MD, Pollina L, Mosca F, Peters G, Campani D and Boggi U: Molecular mechanisms underlying the synergistic interaction of the novel anticancer drug celandine with gemcitabine in preclinical models of pancreatic cancer. Polski Tygodnik Lekarski 11: 23-40, 2011.
34. Livak KJ and Schmittgen TD: Analysis of relative gene expression data using real-time quantitative PCR and the 2(-Delta Delta C(T)) method. Methods 25: 402-408, 2001.

35. Sun K, Liu ZS and Sun Q: Role of mitochondria in cell apoptosis during hepaticischemia-reperfusion injury and protective effect of ischemic postconditioning. World J Gastroenterol 10: 1934-1938, 2004

36. Zhang L, Li C, Quan R and Xie S: The effect of electroacupuncture on neuronal apoptosis and related functions in rats with acute spinal cord injury. Chin Med 5: 199-210, 2014.

37. Radak Z, Zhao Z, Koltai E, Ohno H and Atalay M: Oxygen consumption and usage during physical exercise: The balance between oxidative stress and ROS-dependent adaptive signaling. Antioxid Redox Signal 18: 1208-1246, 2013.

38. Cardoso AR, Kakimoto PA and Kowaltowski AJ: Diet-sensitive sources of reactive oxygen species in liver mitochondria: Role of very long chain Acyl-CoA dehydrogenases. PLoS One 8: e77088, 2013.

39. Reuter S, Gupta SC, Chaturvedi MM and Aggarwal BB: Oxidative stress, inflammation, and cancer: How are they linked? Free Radic Biol Med 49: 1603-1616, 2010.

40. Chao DT and Korsmeyer SJ: BCL-2 family: Regulators of cell death. Annu Rev Immunol 16: 395-419, 1998

41. Oltvai ZN, Milliman CL and Korsmeyer SJ: Bcl-2 heterodimerizes in vivo with a conserved homolog, Bax, that accelerates programmed cell death. Cell 74: 609-619, 1993.

42. Klune JR and Tsung A: Molecular biology of liver ischemia/ reperfusion injury: Established mechanisms and recent advancements. Surg Clin North Am 90: 665-677, 2010.

43. Weigand K, Brost S, Steinebrunner N, Büchler M, Schemmer P and Müller M: Ischemia/reperfusion injury in liver surgery and transplantation: Pathophysiology. HPB Surg 2012: 176723 , 2012.

44. Marecki H, Bozorgzadeh A, Porte RJ, Leuvenink HG, Uygun K and Martins PN: Liver ex situ machine perfusion preservation: A review of the methodology and results of large animal studies and clinical trials. Liver Transpl 23: 679-695, 2017.

45. Montalvo-Jave EE, Escalante-Tattersfield T, Ortega-Salgado JA, Piña E and Geller DA: Factors in the pathophysiology of the liver ischemia-reperfusion injury. J Surg Res 147: 153-159, 2008.

46. Kaczorowski DJ, Tsung A and Billiar TR: Innate immune mechanisms in ischemia/reperfusion. Front Biosci (Elite Ed) 1: 91-98, 2009.

47. Zeng XP, Li XJ, Zhang QY, Liu QW, Li L, Xiong Y, He CX, Wang YF and Ye QF: Tert-Butylhydroquinone protects liver against ischemia/reperfusion injury in rats through Nrf2-activating anti-oxidative activity. Transplant Proc 49: 366-372, 2017.

48. Tao X, Sun X, Xu L, Yin L, Han X, Qi Y, Xu Y, Zhao Y, Wang C and Peng J: Total flavonoids from Rosa laevigata Michx fruit ameliorates hepatic ischemia/reperfusion injury through inhibition of oxidative stress and inflammation in rats. Nutrients 8: E418, 2016

49. Chou YH, Ho FM, Liu DZ, Lin SY, Tsai LH, Chen CH, Ho YS, Hung LF and Liang YC: The possible role of heat shock factor-1 in the negative regulation of heme oxygenase-1. Int J Biochem Cell Biol 37: 604-615, 2005.

50. Pastukh V, Ruchko M, Gorodnya O, Wilson GL and Gillespie MN: Sequence-specific oxidative base modifications in hypoxiainducible genes. Free Radic Biol Med 43: 1616-1626, 2007.

51. Hsieh CY, Hsiao HY, Wu WY, Liu CA, Tsai YC, Chao YJ, Wang DL and Hsieh HJ: Regulation of shear-induced nuclear translocation of the Nrf2 transcription factor in endothelial cells. J Biomed Sci 16: 12, 2009.

52. St Peter SD, Imber CJ and Friend PJ: Liver and kidney preservation by perfusion. Lancet 359: 604-613, 2002.

This work is licensed under a Creative Commons Attribution-NonCommercial-NoDerivatives 4.0 International (CC BY-NC-ND 4.0) License. 